\title{
Redesigning OP2 Compiler to Use HPX Runtime Asynchronous Techniques
}

\author{
Zahra Khatami $^{1,2}$, Hartmut Kaiser ${ }^{1,2}$, and J. Ramanujam ${ }^{1}$ \\ ${ }^{1}$ Center for Computation and Technology, Louisiana State University \\ ${ }^{2}$ The STE ||AR Group, http://stellar-group.org
}

\begin{abstract}
Maximizing parallelism level in applications can be achieved by minimizing overheads due to load imbalances and waiting time due to memory latencies. Compiler optimization is one of the most effective solutions to tackle this problem. The compiler is able to detect the data dependencies in an application and is able to analyze the specific sections of code for parallelization potential. However, all of these techniques provided with a compiler are usually applied at compile time, so they rely on static analysis, which is insufficient for achieving maximum parallelism and producing desired application scalability. One solution to address this challenge is the use of runtime methods. This strategy can be implemented by delaying certain amount of code analysis to be done at runtime.
\end{abstract}

In this research, we improve the parallel application performance generated by the OP2 compiler by leveraging HPX, a $\mathrm{C}++$ runtime system, to provide runtime optimizations. These optimizations include asynchronous tasking, loop interleaving, dynamic chunk sizing, and data prefetching. The results of the research were evaluated using an Airfoil application which showed a $40-50 \%$ improvement in parallel performance.

Index Terms-HPX, OP2, Asynchronous Task Execution, Interleaving Loops, Controlling Chunk Sizes, Prefetching Data.

\section{INTRODUCTION}

Unstructured grids are well studied and utilized in various application domains. OP2 provides a framework for the parallel execution of these unstructured grid applications on different multi-core/many-core hardware architectures [1], [2]. The main goal of developing OP2 is to provide an abstraction level for users to parallelize their applications without having to worrying about architecture specific optimizations. This allows scientists to invest most of their time in understanding their domain problems, without learning details of new architectures, and still achieve efficient utilization of the available hardware. The framework is designed to achieve the nearoptimal scaling on multi-core processors [3], [4]. However, as the compiler only has a static and defined access pattern [5], [6], [7], its analysis is not enough to obtain desired parallel scalability. In order to reach this goal, OP2 needs to be able to extract parallelism automatically at runtime.

In this research, we propose different optimization methods that provide dynamic information for code generated by the OP2 compiler, including providing asynchronous task execution, interleaving different loops together, dynamically setting chunk sizes of different dependent loops based on each other, and prefetching data. These proposed techniques are implemented using HPX runtime system via redesigning the OP2 framework in a way that employs both compiler's static analysis and dynamic runtime information. HPX is a parallel $\mathrm{C}++$ runtime system that facilitates distributed operations and enables fine-grained task parallelism resulting in a better load balancing [8], [9]. It provides an efficient scalable parallelism by significantly reducing processor starvation and effective latencies while controlling overheads [10].

A closer analysis of unstructured applications reveals that synchronization is only required between small tasks. Prevalent parallelization paradigms, however, coerce users to join all tasks together before proceeding to the next step in the application. In HPX, we can utilize the future construct to allow every task to proceed as long as the values it depends on are ready [11]. This feature allows the HPX to relax the global barriers, enable flexibility, and improve the parallel performance of applications. In this research, HPX uses futures based techniques to develop a new task execution strategy for codes generated by the OP2 compiler which is the basis for asynchronous tasking and interleaving loops.

In order to control the overheads introduced by the creation of each task, it is important to control the amount of work performed by each task. This amount of work is known as the chunk size [11], [12]. In addition, to properly interleave loops it is important for each loop to have very similar execution times which allows the waiting time between the execution of each loop to be minimal. We propose to address these two obstacles by creating a new execution policy which will dynamically control the chunk sizes during the application's execution. In addition, we also propose to create a new cache prefetcher that aids in prefetching data for each time step to reduce memory accesses latencies. This method is implemented in such a way that data of the next iteration step is prefetched into the cache memory using a prefetching iterator called in each iteration within a loop. The main difference between this method and the other existing methods is that HPX implementation combines a thread based prefetching method with the asynchronous task execution, which results in having asynchronous execution while prefetching data of all the containers within a loop.

To our knowledge, we present a first attempt of redesigning OP2 to utilize the runtime techniques for improving performance of the parallel unstructured grid applications. The combination of these proposed techniques should yield a more portable and performant software stack for unstructured grid applications and enable the applications to properly scale to a higher level of parallelism compared to the existing OP2 
implementation. The results evaluated in Section VI show that the parallelization performances are improved by around $40-50 \%$ for an Airfoil application. The remainder of this paper is structured as follows: Section II briefly introduces OP2; Section III introduces a dataflow object in HPX; Section IV shows the details of the dataflow implementation with the new execution policy within OP2; Section $\mathrm{V}$ presents the prefetching method implemented in one of the HPX parallel algorithms, and Section VI evaluates the the scaling speedup of the experimental tests. The conclusions and the future works can be found in Section VII

\section{OP2}

OP2 is an active library that provides a parallel execution framework for unstructured grid applications on different multi-core/many-core hardware architectures [1]. It utilizes a source-to-source translator for generating code which targets different hardware configurations [2], [3], [13]. The code can be transformed easily into different configurations such as serial, multi-threaded using OpenMP and CUDA, or heterogeneous which utilizes MPI, OpenMP, and CUDA [3]. In this section, we first walk through a simple OP2 code to show its implementation details and then we introduce the Airfoil application which is used as a case study for this research.

\section{A. Simple Code Implementation with OP2}

This section generally shows how unstructured grids are defined with OP2. The OP2 API handles the data dependencies by providing mesh represented data layouts. The provided framework is defined based on sets, data on sets, mapping connectivity between the sets, and the computation on each set [2], [14]. Sets can be nodes, edges or faces. In these unstructured grids, the connectivity information is used to specify different mesh topologies. Figure 1 shows a mesh example that includes nodes and faces as sets. The value of data associated with each set is shown below each set and the mesh is represented by the connections between them.

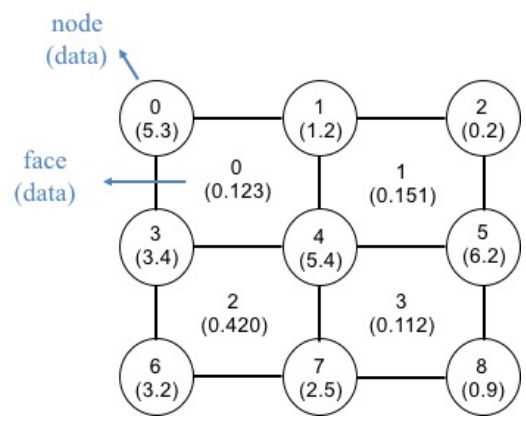

Fig. 1: The mesh represented data layouts provided with OP2.

OP2 API for the mesh in figure 1 is shown as follows, which is the $\mathrm{C} / \mathrm{C}++\mathrm{API}$ and defines 12 edges and 9 nodes:

op_set nodes;

op_decl_set (9, nodes, "nodes");

op_set edges ;

op_decl_set (12, edges, "edges");
The mapping that declares the connection between 2 nodes is defined as follow:

int edge_map $[28]=\{0,1,1,2,2,5,5,4,4,3,3,6,6,7$,

$7,8,0,3,1,4,2,5,3,6,4,7,5,8\}$

op_map pedge ;

op_decl_map (edges, nodes,2, edge_map, pedge, "pedge")

op_decl_map shows that each edge is mapped on two different nodes. The values of each node and face are assigned as follow:

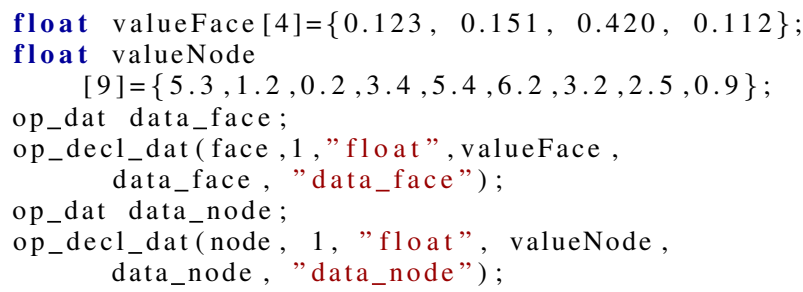

These sets and meshes are used to define a loop over a given set. The more details about OP2 design and performance analysis can be found in [1] and [14], which shows that all unstructured grid applications can be easily described with sets and meshes as shown in the above example. These methods place no restriction on the algorithm and they allow the programmer to choose unique operations on each set.

\section{B. Airfoil Application}

In this research, we study an Airfoil application, which is a standard unstructured mesh finite volume computational fluid dynamics (CFD) code, presented in [15], for the turbomachinery simulation and consists of over $720 \mathrm{~K}$ nodes and about 1.5 million edges. As described in [15] and [16], it consists of five parallel loops: op_par_loop_save_soln, op_par_loop_adt_calc, $o p \_p a r \_l o o p \_r e s \_c a l c, \quad o p \_p a r \_l o o p \_b r e s \_c a l c$, op_par_loop_update, shown in figure 2. All of the computations on each set are implemented within these loops by performing operations of the user's kernels defined in a header file for each loop: save_soln.h, adt_calc.h, res_calc.h, bres_calc.h and update.h. Each argument passed to each loop is generated based on data values used with $o p \_a r g \_d a t$.

Figure 3 demonstrates $o p \_p a r \_l o o p \_s a v e \_s o l n$ that applies save_soln on cells based on the arguments generated with $o p \_a r g \_d a t$ using $p \_q$ and $p \_q o l d$ data values. The function $o p \_a r g \_d a t$ creates an OP2 argument based on the information passed to it. These arguments explicitly indicate that how each of the underlying data can be accessed inside a loop: $O P \_R E A D$ (read only), OP_WRITE (write) or OP_INC (increment to avoid race conditions due to indirect data access) [1]. More details can be found in [2] and [13].

The loop parsed with OP2 in figure 4 illustrates how each cell updates its data value by accessing blockId, offset_b, and nelem data elements. The arguments are passed to the save_soln user kernel subroutine, which does the computation for each iteration of an inner loop from offset $b$ to 


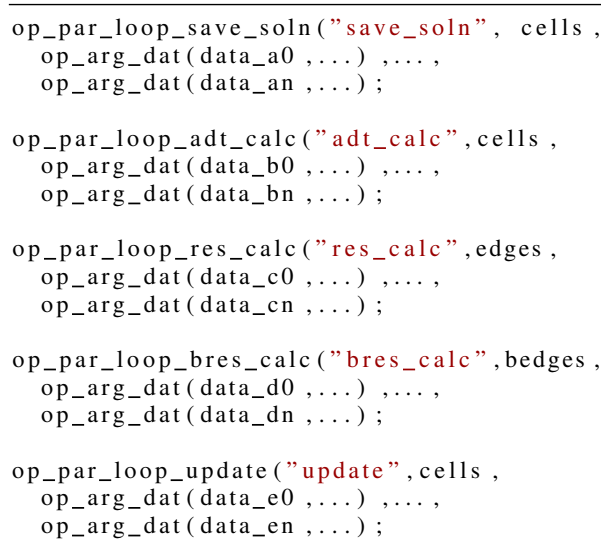

Fig. 2: Five loops used in Airfoil.cpp for saving old data values, applying computation, and updating each data value.

op_par_loop_save_soln("save_soln", cells

op_arg_dat ( $\bar{p}_{-} q,-\overline{1}$, OP_ID $_{4} 4$, , d ouble", OP_READ),

op_arg_dat (p_qold, -1, OP_ID, 4, , double ",OP_WRITE) ) ;

Fig. 3: op_par_loop_save_soln represents one of the loops used in an Airfoil application.

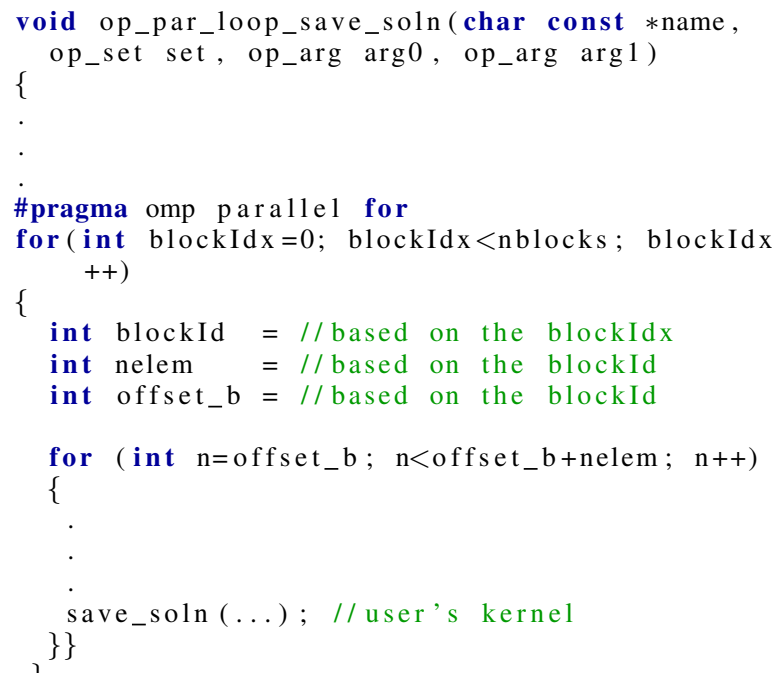

Fig. 4: \#pragma omp parallel for is used for a loop parallelization for an Airfoil application. offset_b+nelem of each iteration of an outer loop from 0 to nblocks. Also it illustrates that OpenMP is used for the parallel processing within a node. It is important to note that the outputs of the computations shown in figure 2 cannot be passed to the outside of the loop, therefore, the current OP2 design doesn't provide a method for interleaving loops together. This creates implicit global barrier after each loop as the threads inside the loop must wait to synchronize before exiting the loop [17]. Barriers, naturally, impede optimal parallelization by causing the parallel threads and processes to wait. In order to solve this problem, this research sets out to optimize the performance of code generated by the OP2 compiler using the HPX runtime. The source-to-source code translator of OP2 is written in Matlab and Python [13]. In this research, its Python source-to-source code translator is modified to automatically generate the parallel loops using HPX library calls.

\section{HPX}

In this research different dynamic optimizations are proposed for improving the performance of code generated by the OP2 compiler that are implemented using HPX runtime system, which has been developed to overcome limitations such as global barriers and poor latency hiding [9], [10] by embracing new ways of coordinating parallel execution, controlling synchronization, and implementing latency hiding utilizing Local Control Objects (LCO) [16], [18]. These objects have the ability to create, resume, or suspend a thread when triggered by one or more events. LCOs provide traditional concurrency control mechanisms such as various types of mutexes, semaphores, spinlocks, condition variables and barriers in HPX. These objects improve the efficiency of an application by permitting highly dynamic flow control as they organize the execution flow, omit global barriers, and enable thread execution to proceed as far as possible without waiting. More details about LCO design and its performance can be found in [10], [19], [20].

The two implementations of LCOs most relevant to this research are the future construct and the dataflow template. HPX provides a multi-threaded, message-driven, split-phase transaction, and distributed shared memory programming model using futures and dataflow based synchronization on the large distributed system architectures, which are explained in the following sections.

\section{A. Future}

future is a computational result that is initially unknown but becomes available at a later time [11]. The goal of using future is to let every computation proceed as far as possible. Using future enables threads to continue their executions without waiting for the results of the previous steps to be completed, which eliminates the implicit global barrier at the end of the execution of an OpenMP parallel loop. future based parallelization provides the rich semantics for exploiting higher level parallelism available within each application that may significantly improve its scalability. 


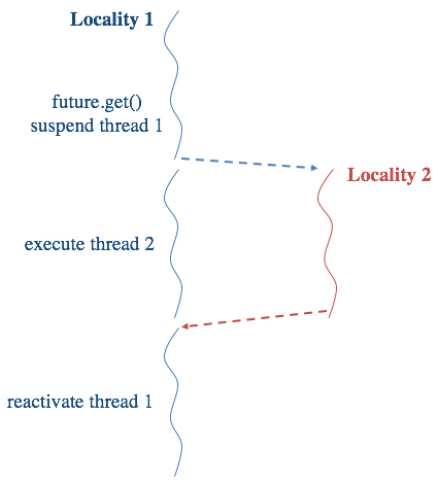

Fig. 5: The principle of the operation of the future in HPX. Thread 1 is suspended only if the results from locality 2 are not readily available. Thread 1 accesses the future value by performing future.get (). If results are available, Tread 1 continues to complete the execution.

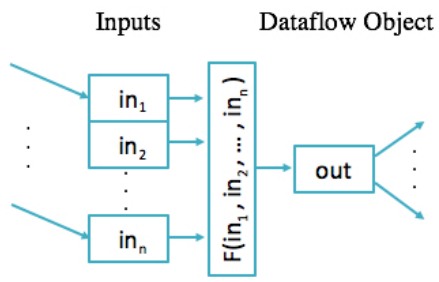

Fig. 6: A dataflow object encapsulates a function $F\left(i n_{1}, i n_{2}, \ldots, i n_{n}\right)$ with $n$ inputs from different data resources. As soon as the last input argument has been received, the function $F$ is scheduled for an execution.

Figure 5 shows the scheme of the future performance with 2 localities, where a locality is a collection of processing units (PUs) that have access to the same main memory. It illustrates that the other threads do not stop their progress even if the thread, which waits for the value to be computed, is suspended. Threads access a future value by performing future.get (). When the result becomes available, the future resumes all HPX suspended threads waiting for that value. It can be seen that this process eliminates the global barrier synchronizations, as only those threads that depend on the future value are suspended. With this scheme, HPX allows asynchronous execution of the threads.

\section{B. Dataflow Object}

dataflow object provides a powerful mechanism for managing data dependencies without the use of global barriers [21], [8]. Figure 6 shows the schematic of a dataflow object, which encapsulates a function $F\left(i n_{1}, i n_{2}, \ldots, i n_{n}\right)$ with $n$ future or non-future inputs from different data resources. If an input is a future, then the invocation of the function will be delayed. Non-future inputs are passed through. A dataflow object waits for a set of futures to become ready and as soon as the last input argument has been received, the function $F$ is scheduled for the execution [19]. Because the dataflow object

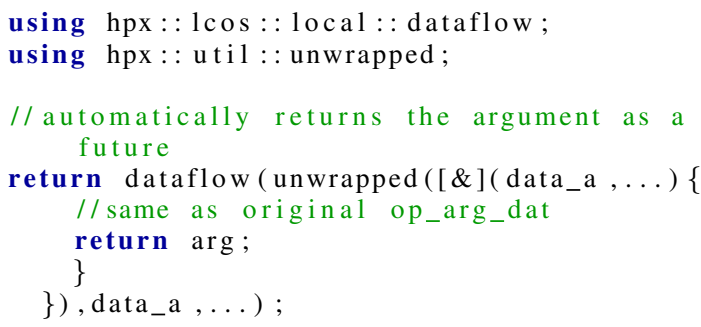

Fig. 7: op_arg_dat is modified to create an argument as a future that is passed to a function through op_par_loop shown in figure 2

returns a future, its result can be fed to other objects in the system including other dataflows. These chained futures, by their nature, represent a dependency tree that automatically generates an execution graph. This graph is executed by the runtime system as each nodes dependencies are meet. As a result, dataflow minimizes the total synchronization by scheduling new tasks as soon as they can be run instead of waiting for entire blocks of tasks to finish computation.

\section{IMPLEMENTING DATAFLOW IN OP2}

In this section, the new method is proposed for parallelizing loops generated with OP2, which is based on dataflow implementation that solves the current challenges of OP2. In this method, the OP2 API is modified in such a way that op_arg_dat used in each loop in figure 2 produces an argument as a future for dataflow object inputs. Figure 7 shows the modified op_arg_dat, in which data_a,... expressed at the last line of the code invokes a function only once all of them get ready. unwrapped is a helper function in HPX, which unwraps the futures and passes along the actual results. This implementation also generates an output argument as a future and as a result, all of the arguments of each loop in figure 2 are passed as a future to the kernel function through op_par_loop.

\section{A. Parallelizing Loops Using for_each}

Parallelizing loops and controlling chunk sizes are implemented by using for_each algorithm and persistent_auto_chunk_size as an execution_policy respectively. In figure 8, dataflow is implemented with for_each for the loop in figure 4, that aids to parallelize the outer loop.for_each is one of the HPX parallel algorithms that is able to automatically control the chunk size during the execution by determining number of the iterations to be run on each HPX thread. Moreover, HPX is able to execute loops in sequential or in parallel by applying execution_policies, which are briefly described in Table [19]. The concept of the execution_policy developed in HPX is used to specify the execution restrictions of the work items, in which calling with a sequential execution policy makes the algorithm to be run sequentially and calling with a parallel execution policy allows the algorithm to be run in parallel [18]. 


\begin{tabular}{|c|c|c|}
\hline Policy & Description & Implemented by \\
\hline seq & sequential execution & Parallelism TS, HPX \\
\hline par & parallel execution & Parallelism TS, HPX \\
\hline par_vec & $\begin{array}{l}\text { parallel and } \\
\text { vectorized execution }\end{array}$ & Parallelism TS \\
\hline seq(task) & $\begin{array}{l}\text { sequential and } \\
\text { asynchronous execution }\end{array}$ & HPX \\
\hline par(task) & $\begin{array}{l}\text { parallel and } \\
\text { asynchronous execution }\end{array}$ & HPX \\
\hline
\end{tabular}

TABLE I: The execution policies implemented in HPX.

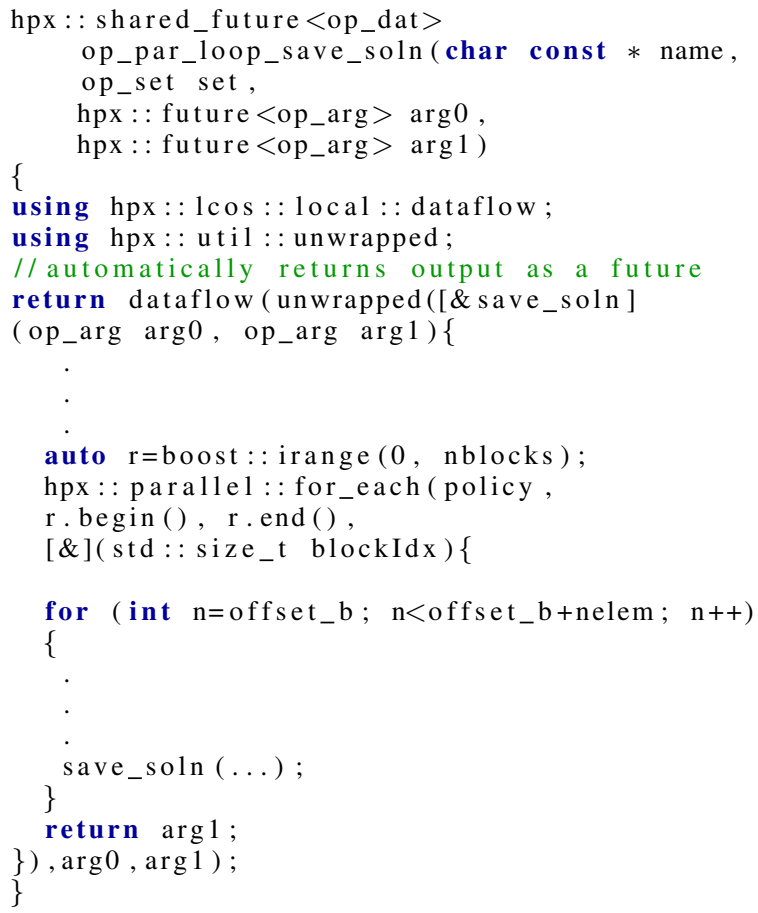

Fig. 8: Implementing for_each within dataflow for the loop parallelization in OP2 for the loop in figure 4 It makes the invocation of a loop asynchronous by returning output as a future. dataflow allows automatically creating execution graph, which represents a dependency tree.

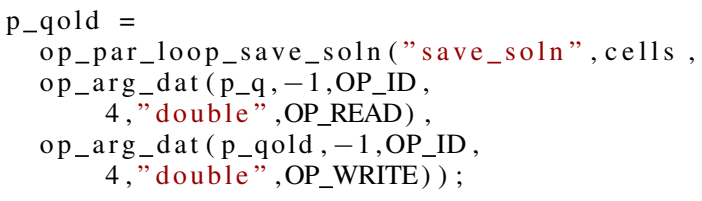

Fig. 9: Airfoil.cpp is changed while using dataflow for the loop parallelization in OP2. p_qold is returned as a future from each kernel function after calling op_par_loop.

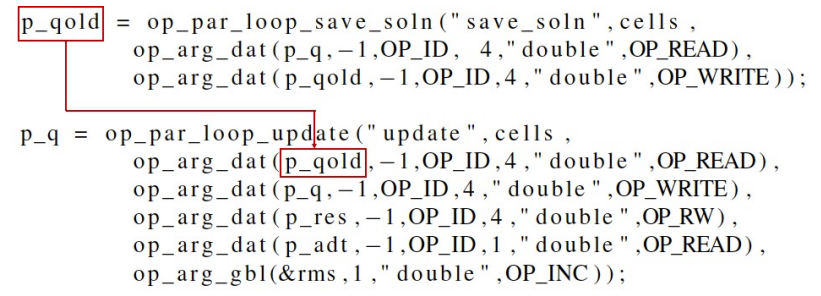

Fig. 10: The proposed method makes OP2 able to interleave thses two loops together by passing p_qold output of op_par_loop_save_soln as an input argument for op_par_loop_update.

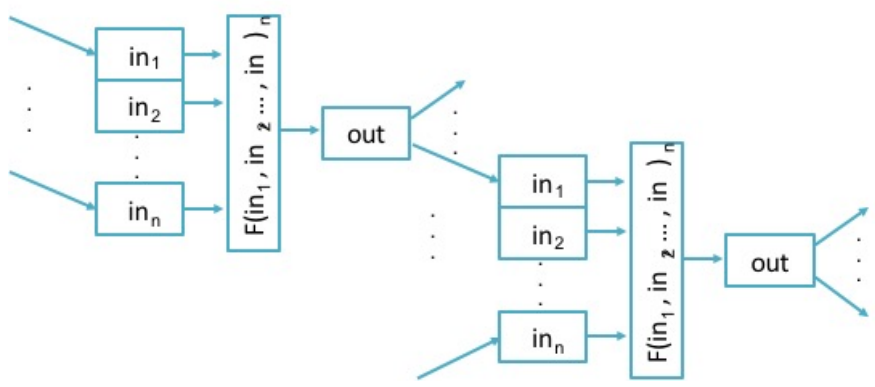

Fig. 11: dataflow provides a way for interleaving execution of different loops together by generating output as a future and passing all inputs as futures as well.

Figure 8 also illustrates that $\arg 0$ and $\arg 1$, which are created as a future with op_arg_dat using $p \_q$ and $p \_q o l d$ respectively, are passed as a future within a loop. This loop will be executed only if these arguments get ready. Then, the output argument, which is arg1 in this example, is passed as a future to the outside of the loop and it is stored within $p \_q o l d$ shown in figure 9. This method is implemented to all of the loops in figure 2, and as a result, each kernel function returns an output argument as a future. The loop execution may depend on the results of the other previous loops. So by this method, the results of the loops can be passed as future inputs to the other loops, which makes OP2 able to interleave different loops. For example, $p \_q o l d$ value updated in $o p \_p a r \_l o o p \_s a v e \_s o l n$ is used as an input argument for op_par_loop_update as shown in figure 10, which using this proposed method makes it able to interleave this two loops together by passing output of op_par_loop_save_soln as an input argument for op_par_loop_update.

Figure 11 shows generally that by implementing proposed method, the future output of each loop passed as an input of the other loops makes OP2 able to interleave different loops together at runtime. As a result, if the loops are not dependent on each other, they can be executed without waiting for the previous loops to complete their tasks, however, if they depend on the parameters from the previous loops, they will wait until the previous loops complete their processes. This proposed 

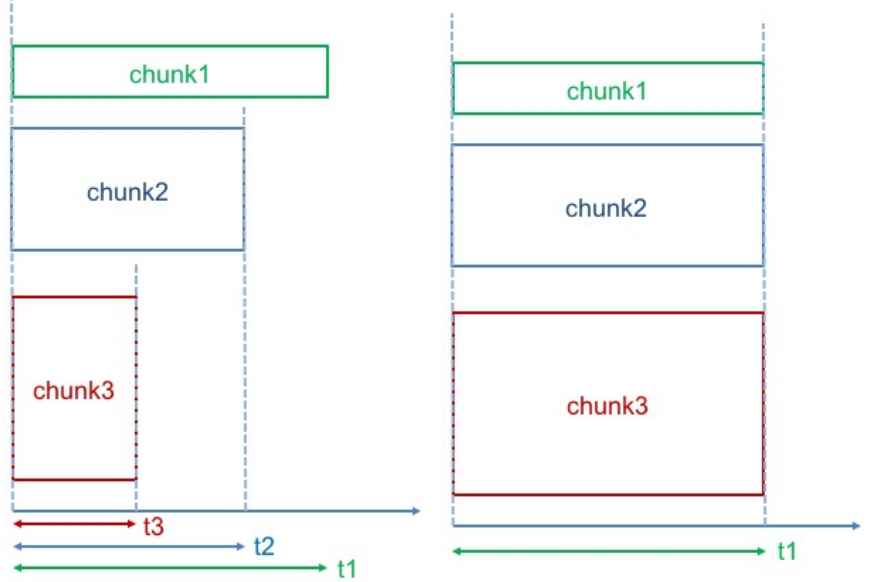

(a) Chunk sizes with different ex- (b) Chunk sizes with the same ecution time

Fig. 12: Setting chunk sizes of different dependent loops based on each other.

method removes the unnecessary barrier synchronizations between different loops and execute them asynchronously.

\section{B. Controlling Chunk Sizes}

As it is explained in section IV-A figure 11 shows how dataflow provides a way of interleaving execution of different loops together. In a case of having dependent loops, the execution of each chunk in a loop depends on the execution of the chunks in the previous loop. By using par as an execution policy, different chunks with different execution time regardless of the chunk sizes of the other loops are determined for each loop shown in figure 12a, which may increase the waiting time between them. So for decreasing this waiting time, the execution time of each chunk in these dependent loops should be the same. For this purpose, the new execution policy is proposed in this section, named persistent_auto_chunk_size, that makes all chunk sizes of different loops having same execution time as shown in figure $12 \mathrm{~b}$. In this policy, the chunk size of the first loop is determined automatically with for_each algorithm. Then the chunk sizes of each second and third loops are determined based on the execution time of the chunk in the first loop. As a result, all chunks of all these three loops will have the same execution time. It should be note that chunk1, chunk2 and chunk3 have different sizes but with the same execution time.

\section{HPX DATA PREFTECHER}

Data prefetching is one of the methods for reducing memory accesses latencies by calling data required for the next step into the cache [22]. The simplest form of the cache prefetching can be implemented by prefetching cache line of the next iteration as soon as the current cache line is referenced [23], [24]. Hardware, software and thread prefetching are different traditional techniques for this purpose.
Various hardware prefetching methods has been proposed that one of them is using one-block-lookahead (OBL) scheme [25]. In this method, the blocks $i+1, i+2, \ldots$, and $i+n$ are prefetched whenever the block $i$ is brought to the cache that results in reducing cache misses significantly. Creating reference prediction table [26], [27] is another method to limit unnecessary prefetching and to predict the future memory references. However, one of the big challenges exists in most of these hardware prefetching methods is that the prefetcher uses the past access pattern by considering data stream, which cannot handle an irregular access pattern.

In the software prefetcher method, the prefetching data is implemented by using prefetch directives in the code. One of the problem of this method is that these prefetching instructions are inserted with programmer or compiler into the applications, which has the high probability of the cache miss occurrences. Another problem is introducing additional overhead for executing these prefetch instructions. There has been many developments proposed for optimizing this technique that mostly are obtained by prefetching pointer-based data structures [25], [28]. Mowry's algorithm [29] is one of the recent prefetching optimization that defines the affine arrayreferences as the prefetching candidates within an inner-most loop, performs the loop unrolling, and creates the multiple memory references within a loop. As a result, the exact missing instance is prefetched, which avoids the unnecessary prefetching and reduces prefetching overheads. Jump pointer prefetching [26], [27] is another proposed software prefetching approach, which is implemented by inserting additional pointers into a dynamic data structure for connecting nonconsecutive elements within a loop. This technique allows prefetching data by creating pointer chain and results in overlapping fetching process of multiple elements simultaneously. However, this technique also has the difficulty in handling sequences of the irregular data accesses [23].

Thread based prefetching method is usually preferred over the software / hardware prefetching methods, since it precomputes the load addresses accurately and it is able to follow more complex patterns compared to the other methods [24]. This technique executes an application in the prefetcher thread context and brings data of the next cache line into the shared cache before the main thread accesses it. However, the scaling can be degraded with this method because of

1) cache misses: the prefetcher could make slower progress than the main thread, and

2) global barriers: a global barrier is needed to synchronize the prefetcher with the main thread [22], [24], [25].

In this section, the new prefetching method is introduced in HPX that combines a thread based prefetching with an asynchronous task execution. The main goal of this method is not only to reduce the memory accesses latencies, but also to relax the global barriers, which results in a better parallel performance.

Figure 13 shows the scheme of using future and the proposed prefetching iterator, which makes HPX to have the asynchronous execution while prefetching data of all the 


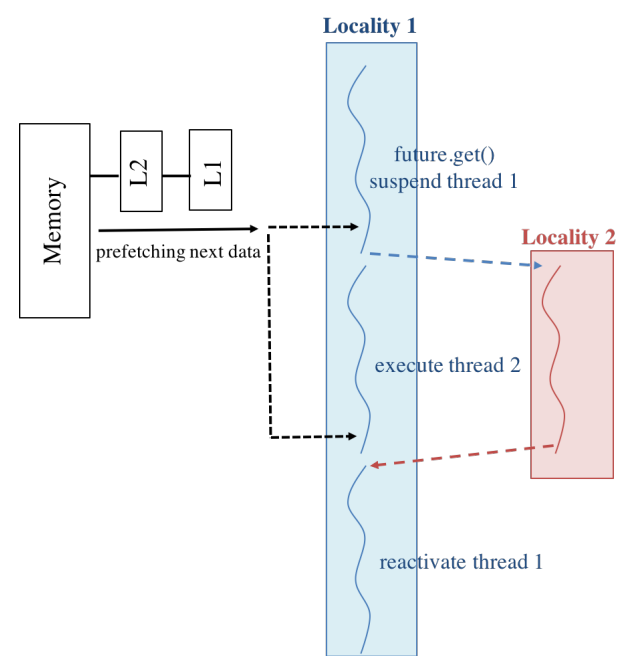

Fig. 13: Data of the next iteration step is prefetched into the cache memory with the prefetching iterator called in each iteration within the for_each

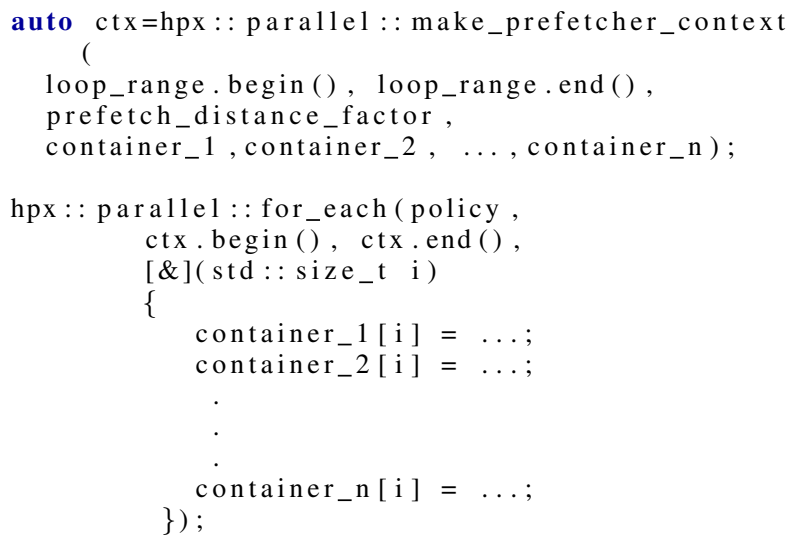

Fig. 14: The prefetching method used in for_each. The prefetching iterator in for_each is called by using ctx_begin, which is the struct that references to all container in the loop.

containers within a loop of the next step in to the cache memory in each iteration. Moreover, HPX is able to prefetch data in sequential or in parallel with applying execution_policy described in Table I This method is added to the method explained in section IV-A to decrease the memory access latencies while parallelizing loops.

Figure 14 shows the details of the prefetching method implementation within for_each. The program execution is divided into several chunks within for_each and its iterator is developed to prefetch data of the next chunk size in either sequential or in parallel. The prefetching iterator is initialized with calling constructer of make_prefetcher_context and it is executed by using ctx.begin(), which is the struct that references to all containers used in a loop and loop_range is the range, in which the loop is executed. One of the feature

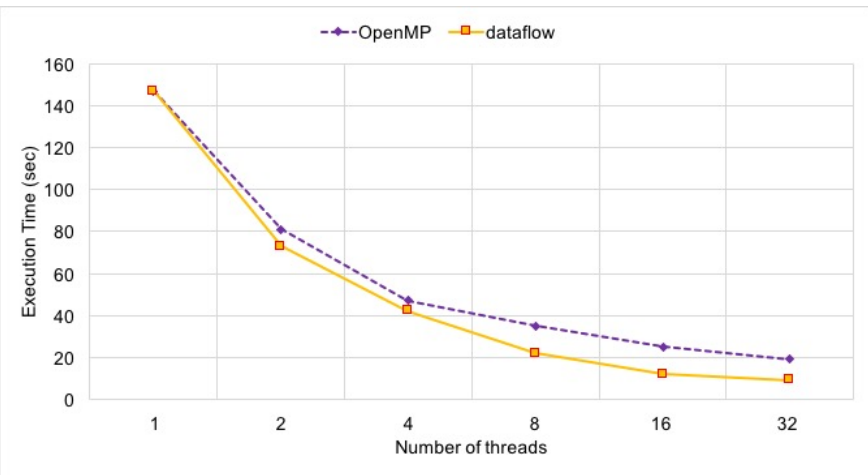

Fig. 15: Comparison results of the execution time between dataflow and \#pragma omp parallel for used for an Airfoil application.

of this prefetcher is that it works with any data types even in a case of having different type for each container. The distance between each two prefetching operations is computed based on the value of prefetch_distance_factor. In order to increase the effectiveness of the prefetcher and to decrease the relative cost, prefetch_distance factor is designed to be determined based on the length of the cache line. As a result, within each prefetcher distance, data of all containers of the next time step are prefetched in each iteration by calling this prefetching iterator. The experimental results of optimizing OP2 performance with HPX discussed in this paper are presented in the next section.

\section{EXPERIMENTAL RESULTS}

In this section, we evaluate the experimental results of our work by comparing our proposed framework to OP2's current design. The main goal of this section is to illustrate that dynamic information obtained at runtime and static information obtained at compile time are both necessary to provide sufficient optimizations for optimal performance. The proposed methods studied in the previous sections are evaluated here. The experiments are executed on the test machine with two Intel Xeon E5-2630 processors, each with 8 cores clocked at 2.4GHZ and 65GB. Hyper-threading is enabled. The OS used by the shared memory system is 32 bit Linux Mint 17.2. and HPX 0.9.99 is used here.

\section{A. Asynchronous Task Execution Provided with Dataflow}

Figure 15 shows the execution time of an Airfoil application using \#pragma omp parallel for and dataflow, which illustrates that HPX and OpenMP has approximately the same performance on 1 thread. We are however able to improve parallel performance in using dataflow for more number of threads. For the speedup analysis, we use strong scaling, for which the problem size is kept the same as the number of cores are increased. Figure 16 shows the strong scaling comparison results that illustrates a $33 \%$ better performance for dataflow due to the asynchronous task execution, the use of futures, and interleaving different dependent loops together. As described in section III dataflow automatically generated 


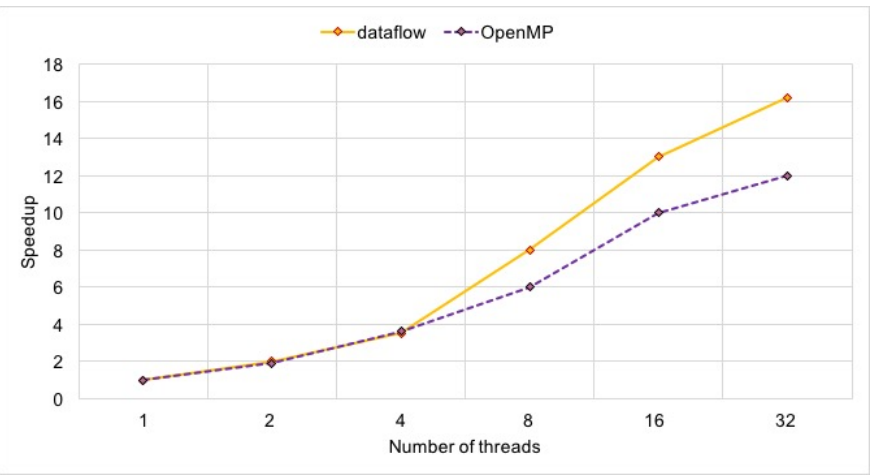

Fig. 16: Comparison results of the strong scaling between dataflow and \#pragma omp parallel for used for an Airfoil application. This comparison result illustrates a better performance for dataflow for a larger number of threads, which is due to the asynchronous task execution. dataflow automatically generates an execution tree, which represents a dependency graph and allows an asynchronous execution of the functions. Hyperthreading is enabled after 16 threads.

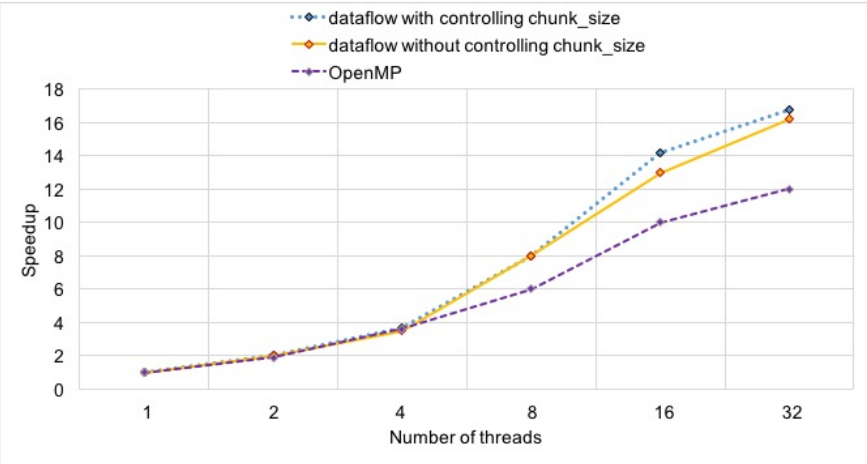

Fig. 17: Comparison results of strong scaling using dataflow with/without setting chunk sizes of different dependent loops based on each other. Hyperthreading is enabled after 16 threads.

an (implicit) execution tree, which represents a dependency graph that results in removing unnecessary global barriers and improving scalability of the parallel applications.

1) Controlling Chunk Sizes: In this section, the chunk sizes of different loops are set by considering chunk sizes determined in the previous loops. Since dataflow enables the compiler to interleave different loops together, the execution of each chunk in each loop depends on the execution of the chunks in the previous loops. So using persistent_auto_chunk_size makes the execution time of each chunks in these loops to be the same, which decreases the waiting time between them. Figure 17 shows the improvement in the performance of dataflow method by using persistent_auto_chunk_size as an execution policy within the loops. For an instance, with 32 threads, the improvement is obtained by about $40 \%$.

For further parallelization performance improvement, data prefetching proposed in section $\mathrm{V}$ is implemented in the dataflow method and its results are evaluated in the next section.

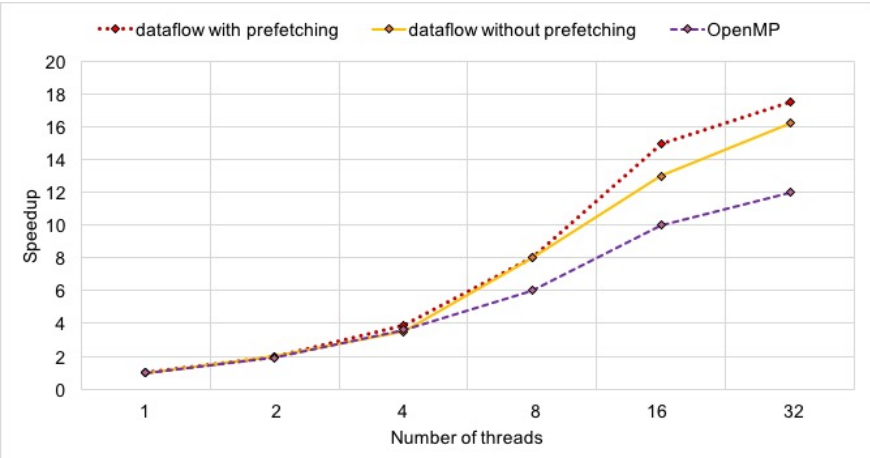

Fig. 18: Comparison results of a dataflow performance by using proposed prefetching method. It shows that the speedup is increased by around $45 \%$ with prefetching data within a loop. Hyperthreading is enabled after 16 threads.

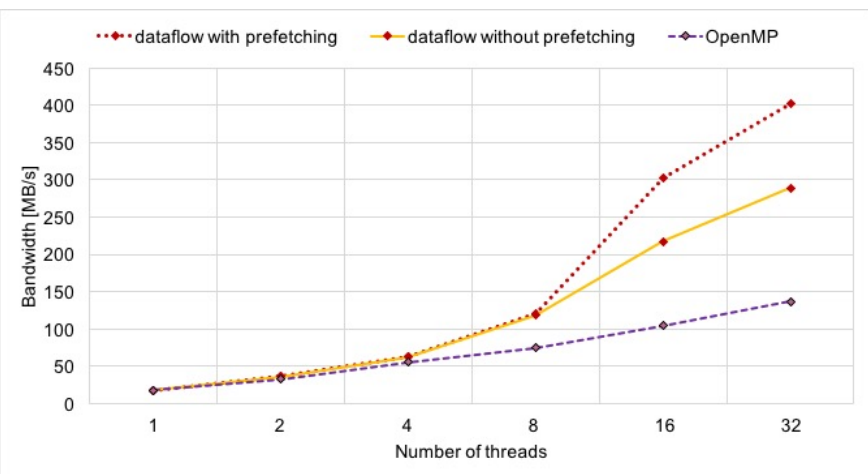

Fig. 19: The data transfer rate of implementing hpx::for_each using standard random access iterator versus prefetching iterator within a dataflow. Hyperthreading is enabled after 16 threads.

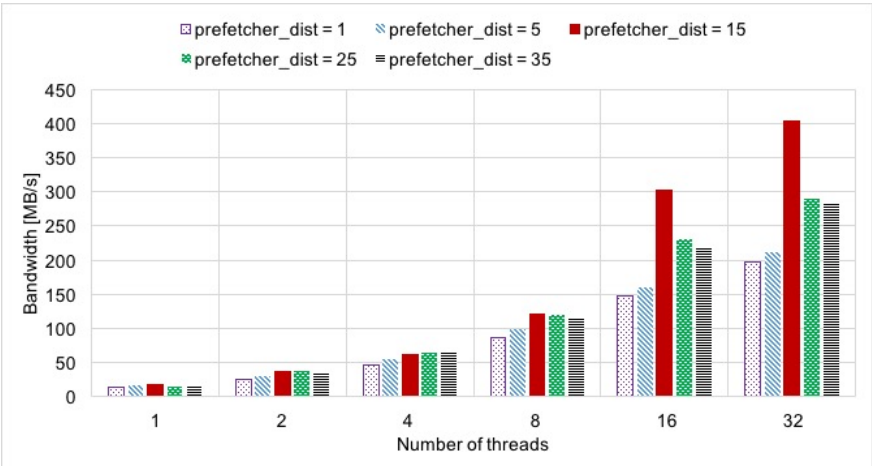

Fig. 20: The data transfer rate of using prefetching iterator for different prefetching distances. Hyperthreading is enabled after 16 threads. 


\section{B. Prefetching Data}

The proposed prefetching method is applied on the dataflow method and its performance is shown in figure 18. This method takes advantage of the asynchronous execution while prefetching data within a loop of the next step in to the cache memory in each iteration step. These results illustrate that the parallel performance of for_each is improved by an average of $45 \%$, which confirms the successful process of avoiding cache misses with implementing HPX prefetcher iterator. The bandwidth rate comparison of these results are also shown in figure 19

The results of the parallel performance of the prefetching iterator measurements with different prefetch_distance_factor are shown in figure 20 It can be seen that for the very large distances, data prefetching cannot improve the parallel performance. On the other hand, very small prefetcher distances causes more data to be prefetched, which becomes more expensive. This cost dominates the gains from prefetching and impedes scaling. It is illustrated that prefetch_distance_factor $=15$ for an Airfoil application improves the parallel performance significantly. These results show the good scalability achieved by HPX and indicates that it has the potential to continue to scale on a larger number of threads.

\section{CONCLUSION}

In this research, we present an implementation of the OP2 compiler that employs HPX runtime techniques to efficiently and automatically parallelize unstructured grid applications to achieve desired parallel scalability. The results illustrate that using both dynamic information provided at runtime and the static information provided at compile time are necessary to obtain a higher parallelism level in the applications.

In the proposed framework, OP2 is able to automatically produce data dependencies based on arguments that are passed into the loops at compile time and, by using HPX parallelism methods, the generated loops can be executed asynchronously. In this framework, we propose different optimization methods that make OP2 execute tasks asynchronously, interleave different loops together, efficiently control the chunk sizes of different dependent loops based on each other, and prefetch data into the cache before its actual access. These proposed methods improved the overall performance of an Airfoil application by $40-50 \%$.

In future research, we plan to improve runtime optimizations with information from the compiler. Since runtime information is often speculative, solely relying on it doesn't guarantee maximizing parallelization performance. In general, the parallelization performance of an application depends on the values measured at runtime and the related transformations such as loop skewing and loop scheduling performed at compile time. Collecting the outcome of the static analysis performed by the compiler could significantly improve the runtime performance.

\section{Acknowledgements}

We would like to thank Adrian Serio from Center for Computation and Technology at Louisiana State University for the invaluable and helpful comments and suggestions to improve the quality of the paper. This works was supported by NSF awards 1447831 .

\section{REFERENCES}

[1] GA Mudalige, MB Giles, I Reguly, C Bertolli, and PHJ Kelly. Op2: An active library framework for solving unstructured mesh-based applications on multi-core and many-core architectures. In Innovative Parallel Computing (InPar), 2012, pages 1-12. IEEE, 2012.

[2] Mike B Giles, Gihan R Mudalige, Zohirul Sharif, G Markall, and Paul HJ Kelly. Performance analysis and optimization of the op2 framework on many-core architectures. The Computer Journal, page bxr062, 2011.

[3] GR Mudalige, MB Giles, B Spencer, C Bertolli, and IZ Reguly. Designing op2 for gpu architectures. Journal of Parallel and Distributed Computing, 2012.

[4] Carlo Bertolli, Adam Betts, Paul HJ Kelly, Gihan R Mudalige, and Mike B Giles. Mesh independent loop fusion for unstructured mesh applications. In Proceedings of the 9th conference on Computing Frontiers, pages 43-52. ACM, 2012.

[5] Devang Patel and Lawrence Rauchwerger. Implementation issues of loop-level speculative run-time parallelization. In International Conference on Compiler Construction, pages 183-197. Springer, 1999.

[6] Lawrence Rauchwerger, Nancy M Amato, and David A Padua. A scalable method for run-time loop parallelization. International Journal of Parallel Programming, 23(6):537-576, 1995.

[7] Lawrence Rauchwerger, Nancy M Amato, and David A Padua. Runtime methods for parallelizing partially parallel loops. In Proceedings of the 9th international conference on Supercomputing, pages 137-146. ACM, 1995.

[8] Thomas Heller, Hartmut Kaiser, Andreas Schäfer, and Dietmar Fey. Using HPX and LibGeoDecomp for scaling HPC applications on heterogeneous supercomputers. In Proceedings of the Workshop on Latest Advances in Scalable Algorithms for Large-Scale Systems, page 1. ACM, 2013.

[9] T Heller, H Kaiser, and Klaus Iglberger. Application of the ParalleX execution model to stencil-based problems. Computer Science-Research and Development, 28(2-3):253-261, 2013.

[10] Patricia Grubel, Hartmut Kaiser, Jeanine Cook, and Adrian Serio. The Performance Implication of Task Size for Applications on the HPX Runtime System. In Cluster Computing (CLUSTER), 2015 IEEE International Conference on, pages 682-689. IEEE, 2015.

[11] Henry C Baker Jr and Carl Hewitt. The incremental garbage collection of processes. In ACM Sigplan Notices, volume 12, pages 55-59. ACM, 1977.

[12] Hartmut Kaiser, Thomas Heller, Bryce Adelstein-Lelbach, Adrian Serio, and Dietmar Fey. HPX: A Task Based Programming Model in a Global Address Space. In Proceedings of the 8th International Conference on Partitioned Global Address Space Programming Models, page 6. ACM, 2014.

[13] Carlo Bertolli, Adam Betts, Gihan Mudalige, Mike Giles, and Paul Kelly. Design and performance of the op2 library for unstructured mesh applications. In Euro-Par 2011: Parallel Processing Workshops, pages 191-200. Springer, 2011.

[14] Mike B Giles, Gihan R Mudalige, Z Sharif, G Markall, and Paul HJ Kelly. Performance analysis of the op2 framework on many-core architectures. ACM SIGMETRICS Performance Evaluation Review, 38(4):9-15, 2011.

[15] MB Giles, D Ghate, and MC Duta. Using automatic differentiation for adjoint cfd code development. 2005.

[16] Zahra Khatami, Hartmut Kaiser, and J Ramanujam. Using hpx and op2 for improving parallel scaling performance of unstructured grid applications. In Parallel Processing Workshops (ICPPW), 2016 45th International Conference on, pages 190-199. IEEE, 2016.

[17] LA Smith. Mixed mode MPI/OpenMP programming. UK High-End Computing Technology Report, pages 1-25, 2000. 
[18] Thomas Heller, Hartmut Kaiser, Patrick Diehl, Dietmar Fey, and Marc Alexander Schweitzer. Closing the performance gap with modern c++. In International Conference on High Performance Computing, pages 18-31. Springer, 2016.

[19] Hartmut Kaiser, Thomas Heller, Daniel Bourgeois, and Dietmar Fey. Higher-level parallelization for local and distributed asynchronous taskbased programming. In Proceedings of the First International Workshop on Extreme Scale Programming Models and Middleware, pages 29-37. ACM, 2015.

[20] Chirag Dekate. Extreme Scale Parallel NBody Algorithm with Event Driven Constraint Based Execution Model. PhD thesis, Citeseer, 2011.

[21] Patricia Grubel, Hartmut Kaiser, Kevin Huck, and Jeanine Cook. Using intrinsic performance counters to assess efficiency in task-based parallel applications.

[22] Jamison Collins, Suleyman Sair, Brad Calder, and Dean M Tullsen. Pointer cache assisted prefetching. In Proceedings of the 35th annual ACM/IEEE international symposium on Microarchitecture, pages 62-73. IEEE Computer Society Press, 2002.

[23] Ilya Ganusov and Martin Burtscher. Efficient emulation of hardware prefetchers via event-driven helper threading. In Proceedings of the 15th international conference on Parallel architectures and compilation techniques, pages 144-153. ACM, 2006.

[24] Jaejin Lee, Changhee Jung, Daeseob Lim, and Yan Solihin. Prefetching with helper threads for loosely coupled multiprocessor systems. Parallel and Distributed Systems, IEEE Transactions on, 20(9):1309-1324, 2009.

[25] Abdel-Hameed Badawy, Aneesh Aggarwal, Donald Yeung, and ChauWen Tseng. The efficacy of software prefetching and locality optimizations on future memory systems. Journal of Instruction-Level Parallelism, 6(7), 2004

[26] David Callahan, Ken Kennedy, and Allan Porterfield. Software prefetching. In ACM SIGARCH Computer Architecture News, volume 19, pages 40-52. ACM, 1991.

[27] Kavita Bala, M Frans Kaashoek, and William E Weihl. Software prefetching and caching for translation lookaside buffers. In Proceedings of the 1st USENIX conference on Operating Systems Design and Implementation, page 18. USENIX Association, 1994.

[28] Daniel F Zucker, Ruby B Lee, and Michael J Flynn. Hardware and software cache prefetching techniques for mpeg benchmarks. IEEE Transactions on Circuits and Systems for Video Technology, 10(5):782796, 2000.

[29] John L Hennessy and David A Patterson. Computer architecture: a quantitative approach. Elsevier, 2011. 